\title{
IMPACTO DE LA FORMACIÓN \\ PARA EL EMPLEO EN LA CALIDAD \\ DE VIDA DE JÓVENES CON DISCAPACIDAD INTELECTUAL
}

\section{Impact of Employment Training on the Quality of Life of Young People with Intellectual Disabilities}

\author{
Sonia GARIJO GERTRUdiX \\ Fundación A LA PAR \\ sonia.garijo@alapar.org \\ Irene SÁNCHEZ CENTENO \\ Fundación A LA PAR \\ Cruz GonZÁlez Ruiz \\ Fundación A LA PAR \\ Marina SÁNCHEZ Rico \\ Universidad Complutense de Madrid. Departamento de Psicobiología \\ y Metodología en Ciencias del Comportamiento \\ Almudena Martorell Cafranga \\ Fundación A LA PAR
}

Recepción: 25 de septiembre de 2020

Aceptación: 13 de abril de 2021

REsumen: El programa CAMPVS de la Fundación A LA PAR es un recurso formativo orientado al empleo para jóvenes con discapacidad intelectual. En el presente trabajo se analiza la influencia del proyecto formativo en la calidad de vida de su alumnado, así como sobre cada una de las dimensiones que conforman dicho constructo. Se ha realizado un estudio pre-post con sesenta y un participantes. Como resultado principal cabe destacar que la calidad de vida de los estudiantes es significativamente mejor después de haber estudiado en CAMPVS, tanto en el índice total de calidad de vida como en cada una de sus dimensiones. Se discute sobre los cambios en las distintas variables. 
IMPACTO DE LA FORMACIÓN PARA EL EMPLEO EN LA CALIDAD DE VIDA

DE JÓVENES CON DISCAPACIDAD INTELECTUAL

S. GARIJO, I. SÁNCHEZ, C. GONZÁLEZ, M. SÁNCHEZ Y A. MARTORELL

Palabras clave: calidad de vida; discapacidad intelectual; formación para el empleo; empleo.

AвSTRACT: The CAMPVS program from A LA PAR Fundation is an employment-oriented training resource for young people with intellectual disabilities. This paper aims to study the influence of the training project on the quality of life of its students, as well as on each of the dimensions which make up this construct. A pre-post study has been conducted with sixty-one participants. As a main result, it is worth noting that the quality of life of the students is significantly higher after having studied at CAMPVS, both in the total quality of life index and in each of its dimensions. Changes in different variables are discussed.

KEYWORDS: quality of life; intellectual disability; training for employment; employment.

\section{Introducción}

L

AS PERSONAS CON DISCAPACIDAD INTELECTUAL viven en una condición de vulnerabilidad (Palacios y Bariffi, 2007) que puede llevarles a ser expulsados total o cultural, sistemas que determinan la participación de una persona en la sociedad. En la actualidad, el sistema educativo no da una respuesta adaptada a las necesidades y potencialidades de los jóvenes con discapacidad intelectual más allá de sus estudios obligatorios (Palacios y Bariffi, 2007), lo que genera un impacto negativo en su calidad de vida (Morán Suárez et al., 2019).

En los últimos veinte años han proliferado las investigaciones acerca de la calidad de vida en distintos ámbitos, incluido el de discapacidad intelectual (Echeita y Simón, 2014). En este escenario destaca como momento clave la Convención Internacional sobre los Derechos de las Personas con Discapacidad (Macho et al., 2012). Esta asamblea surgió con el propósito de promover, proteger y asegurar el pleno derecho de todas las personas con discapacidad, así como promover el respeto de su dignidad inherente (Palacios y Bariffi, 2007). El aumento en el interés investigador y generador de programas dedicados a mejorar la calidad de vida de esta población ha producido que un constructo que en inicio era muy subjetivo y poco concreto haya alcanzado un alto nivel de precisión en la definición, aplicación y medición (Macho et al., 2012).

El avance en la investigación en calidad de vida ha venido precedido del avance en la conceptualización de la discapacidad, también contemplado en la Convención Internacional sobre los Derechos de las Personas con Discapacidad. La noción que actualmente tenemos sobre discapacidad intelectual ha avanzado desde paradigmas que centraban su atención en las limitaciones del funcionamiento individual (características o rasgos de la persona a menudo referidos como "déficits") a un fenómeno humano que surge en la interacción del individuo con un contexto social determinado. El origen de la discapacidad está en factores orgánicos y sociales, y estos dan lugar

Ediciones Universidad de Salamanca / CC BY-NC-ND

Siglo Cero, vol. 52 (4), 2021, octubre-diciembre, pp. 51-65 
a limitaciones funcionales. De esta manera, la discapacidad no es algo invariable que dependa únicamente de la persona. Es una condición que surge en el escenario compartido de la persona y los apoyos que brinda el medio. De esta manera nos centramos en el papel de los apoyos individualizados y en su efecto para la mejora del funcionamiento individual y la calidad de vida (Gómez y Sabeh, 2001; Schalock, 2013).

La calidad de vida es definida como un constructo multidimensional en el que influyen factores personales y ambientales, tales como la salud, el nivel de vida, las relaciones en la intimidad, el vecindario, el trabajo, la vida familiar, la amistad, la ciudad o lugar de residencia y el estado de la propia nación (Schalock y Verdugo, 2007). Actualmente, se describe que una persona tiene calidad de vida cuando sus necesidades básicas se satisfacen y cuando el medio le brinda oportunidades para perseguir sus propias metas dentro de los contextos personales y sociales principales como son el hogar, la comunidad, la escuela y el trabajo.

En este sentido, Schalock y Verdugo (2007) conceptualizan o definen la calidad de vida como un estado deseado de bienestar personal que tiene propiedades universales y propiedades ligadas a la cultura; tiene componentes objetivos y subjetivos, y está influenciado por características personales y factores ambientales.

En cuanto a su medición, destaca el modelo de los autores Schalock y Verdugo (2007) como uno de los modelos con mayores evidencias de validez en el ámbito internacional (Alonso et al., 2013). Las distintas dimensiones que estos autores tienen en cuenta en la medición de la calidad de vida se resumen en autodeterminación, derechos, bienestar emocional, inclusión social, desarrollo personal, relaciones interpersonales y bienestar material (Schalock y Verdugo, 2007).

Fernández Alcaraz (2013) describe que la calidad de vida de las personas se ve modificada por las experiencias que estas tienen, aunque no de forma continua a lo largo de los años. Personas más jóvenes son más susceptibles a cambios en su calidad de vida, tanto en sentido favorable como desfavorable, en comparación con personas más mayores. Por otro lado, también influye la necesidad de apoyo que tenga la persona (Fernández Alcaraz, 2013). Se conoce que las personas con un mayor grado de discapacidad intelectual son menos reactivas a los programas de intervención sobre este constructo.

La formación y el empleo son dos aspectos cruciales para la calidad de vida de las personas, con discapacidad o sin ella (González Aguilar, 2019). Los programas de formación orientada al empleo tienen una gran importancia en este constructo, ya que acercan a la realidad del empleo a las personas con discapacidad intelectual, permitiendo su desarrollo personal y plan de vida (Tobón, 2008).

Las escasas opciones de formación adaptada a los jóvenes con discapacidad intelectual con carácter postobligatorio (Palacios y Bariffi, 2007) hacen que sean necesarias iniciativas que reduzcan esta vulnerabilidad asegurando una mayor igualdad de oportunidades a la hora de acceder a los hitos propios de la etapa adulta, como la consecución y el mantenimiento de un empleo (Velázquez, 2018).

Los apoyos ofrecidos por las entidades se han ido adaptando a las nuevas conceptualizaciones, y en este contexto surge el proyecto CAMPVS de la Fundación A LA PAR, con el objetivo de dar continuidad a la formación de los jóvenes con discapaci- 
dad intelectual una vez han visto finalizada su educación reglada u obligatoria. Otros programas con algunas características similares ya han mostrado evidencias sobre sus efectos beneficiosos en la población con discapacidad intelectual (García-Sanjuan y Berástegui, 2017; Cabezas y Flórez, 2015; Izuzquiza Gasset et al., 2013).

El proyecto CAMPVS tiene una duración de 3 cursos académicos, con un total de 204 créditos ECTS. El objetivo principal que persigue es la inclusión sociolaboral. Además, se realiza una orientación y asesoramiento integral del alumno de manera individual, como apoyo al proceso de enseñanza y aprendizaje, y desde un enfoque centrado en la persona (Rosete et al., 2014), que pretende la obtención de resultados personales de acuerdo con las capacidades e intereses del alumno. Se nutre de teorías provenientes de la psicología, tales como la Inteligencia Emocional y la Psicología positiva (Hunter, 2008). En CAMPVS se utilizan metodologías basadas en el coaching educativo (Giraldez Hayes y Van Nieuwerburgh, 2016) y se complementan con actividades provenientes de la disciplina de aprendizaje y servicio (Tapia, 2006). CAMPVS utiliza como base la filosofía de la formación profesional dual (Samanes, 2016), en la que la empresa se acerca al alumno como agente formativo. Los perfiles formativos responden a la demanda del mercado laboral y a las vocaciones de nuestros alumnos.

El objetivo general del proyecto es fomentar la inclusión sociolaboral de los jóvenes con discapacidad intelectual facilitando los apoyos necesarios; capacitándoles como sujetos activos de su propia vida, y desarrollando sus habilidades adaptativas en entornos reales, a través de la construcción de puentes de colaboración entre el centro, la familia, la empresa y la comunidad, como agentes participativos en el proceso de enseñanza-aprendizaje.

En cuanto a la metodología, el proyecto CAMPVS cuenta con un modelo propio de intervención y de enfoque formativo (Figura 1).

A continuación, se detallan los aspectos centrales en los distintos ejes. En cuanto a los relacionados con el alumno, el modelo formativo se basa en la individualización de la intervención y en la educación a través de la confianza y la generación de vínculos. El enfoque es el propio del desarrollo integral del alumno, fomentando su empoderamiento en todo momento, y contando con la familia como agente de crecimiento. Los contenidos impartidos se relacionan siempre con un aprendizaje funcional, eminentemente práctico y realizado en entornos reales. El rol del profesor en el aula es el de facilitador del proceso de aprendizaje, estableciendo canales de comunicación fluidos con las personas implicadas en el desarrollo del alumno, el trabajo en equipo y la formación permanente. CAMPVS es un proyecto con un marcado carácter innovador y responsable con su papel sensibilizador sobre la discapacidad intelectual.

El presente estudio pretende analizar cómo CAMPVS, un proyecto de formación postobligatoria para el empleo, cambia la calidad de vida de sus estudiantes con discapacidad intelectual. Se espera una mejoría general de los estudiantes en su calidad de vida, tanto en la medida general como en cada una de sus dimensiones. 


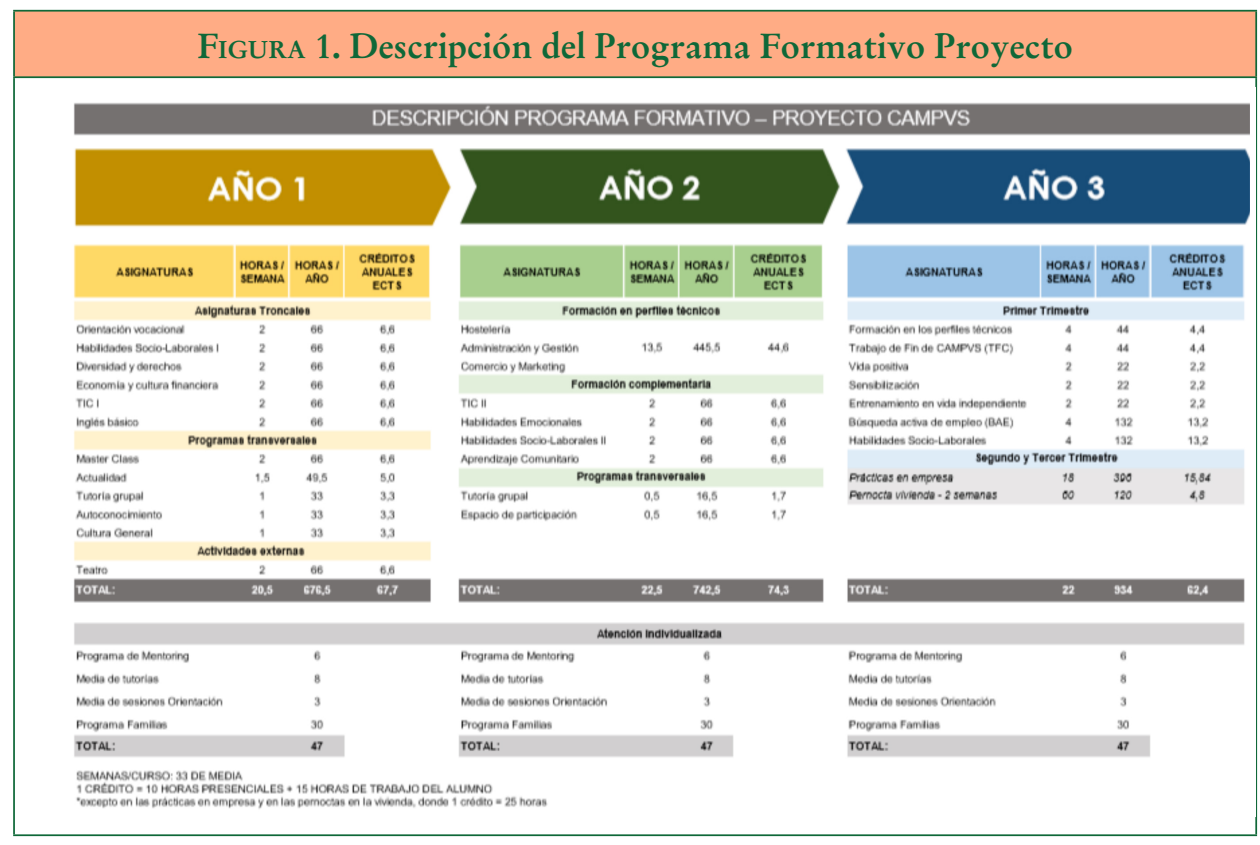

\section{Método}

Se ha realizado un estudio cuasiexperimental con diseño pre-post, donde la variable dependiente es la calidad de vida y la independiente el paso por el proyecto formativo CAMPVS. Se han tomado medidas de forma previa a participar en el proyecto y al finalizar el mismo, siendo la diferencia de tiempo entre una y otra medida de tres años (duración del proyecto).

Todos los participantes dieron su consentimiento para compartir de manera anónima y confidencial su información sobre calidad de vida antes y después del proyecto. Así mismo, todos los procedimientos relacionados con este trabajo se adhirieron a las normas éticas de los comités nacionales e institucionales pertinentes sobre experimentación humana y a la Declaración de Helsinki de 1975, revisada en 2008 (Asociación Médica Mundial, 2008). Así mismo, el presente estudio antes de ser desarrollado fue aprobado por el comité ético de la Fundación A LA PAR.

\subsection{Participantes}

El estudio está formado por 61 adultos jóvenes entre 19 y 32 años (media = 22,56; DT $=2,09$ ), con un grado de discapacidad de entre el $33 \%$ y el $86 \%$ (media $=52,46$ $\% ;$ DT $=14,42)$. De estos 61 sujetos, 37 fueron varones $(60,7 \%)$ y 24 fueron mujeres 
(39,3\%). Todos tienen discapacidad intelectual ligera o límite y pertenecen a las últimas tres promociones consecutivas del proyecto CAMPVS.

Los criterios de admisión para el proyecto formativo CAMPVS son tener discapacidad intelectual con necesidades de apoyo de tipo intermitente o limitado, habilidades adaptativas suficientes para el empleo con apoyo, interés por la formación y el acceso al empleo, una edad comprendida entre los 18 y 30 años y una lectoescritura y manejo numérico funcional. También se tiene en cuenta que la persona se encuentre en un momento personal de estabilidad, que le permita aprovechar el recurso y gozar de una buena salud mental.

Los datos son provenientes de tres promociones consecutivas del programa CAMPVS. La primera muestra corresponde a la segunda promoción del programa y sus datos fueron tomados en 2014 y 2017; la segunda muestra corresponde a la tercera promoción del programa y sus datos fueron tomados en 2015 y 2018, y la tercera muestra se corresponde con la cuarta promoción y sus datos fueron tomados en 2016 y 2019.

\subsection{Instrumento}

El constructo de interés (variable dependiente) es la calidad de vida del alumnado de CAMPVS y su cambio antes y después de pasar por el proyecto formativo.

Se ha utilizado la Escala INICO-FEAPS de Evaluación Integral de la Calidad de Vida de personas con Discapacidad Intelectual o del Desarrollo (Alonso et al., 2013). Las dimensiones estudiadas son Autodeterminación, Derechos, Bienestar emocional, Inclusión social, Desarrollo personal, Relaciones interpersonales, Bienestar material y Bienestar físico. La versión “Autoinforme” consta de los mismos 72 ítems contenidos en la versión anterior, pero esta vez enunciados en primera persona y con un lenguaje más simplificado. El formato de respuesta es en escala tipo Likert de cuatro puntos. Del análisis de las propiedades psicométricas, obtenemos que se trata de un instrumento con una robusta solidez tanto en términos de validez como de fiabilidad. El análisis de la consistencia interna de los ítems se llevó a cabo mediante alfa de Cronbach, donde se obtuvo un coeficiente adecuado: = ,893 (Alonso et al., 2013).

En una entrevista individual se administra el cuestionario de calidad de vida por su tutor de curso. Se utiliza tan solo la versión de autoinforme.

\subsection{Análisis estadístico}

Se ha comprobado si existen diferencias significativas entre las medias para el índice de calidad de vida y sus distintas dimensiones mediante la prueba $t$ de Student para muestras relacionadas cuando se ha cumplido el supuesto de normalidad. En el caso de que no se cumpliese el supuesto de normalidad, se ha comprobado si existen diferencias significativas entre las medianas para el índice de calidad de vida y sus distintas dimensiones mediante la prueba Wilcoxon. 
Por otro lado, también se ha calculado la potencia asociada a cada contraste, siguiendo las recomendaciones de la American Psychological Association (APA Publications and Communications Board Working Group on Journal Article Reporting Standards, 2008) y Castro y Martini (2014). Se ha calculado una $d$ de Coben y el nivel de significación fue fijado a priori en p < 0,05 (Cohen, 2013).

Todos los análisis fueron realizados con SPSS Statistics 27 (2021).

\section{Resultados}

\subsection{Exploración de la muestra}

Los resultados pertenecen a tres promociones consecutivas del proyecto CAMPVS, por lo que se ha realizado un ANOVA de un factor con tres niveles para descartar que existan diferencias entre los grupos en relación con la calidad de vida. Los datos revelan que no existen diferencias significativas entre los tres grupos para el índice de calidad de vida por lo que se ha considerado toda la muestra como una sola $(\mathrm{F}(2,123)=0,529, \mathrm{p}=0,592)$.

Una prueba $t$ de Student para muestras relacionadas mostró que no existían diferencias estadísticamente significativas entre el Cambio en el Índice de Calidad de vida y el género $(\mathrm{T}=0,21 \mathrm{p}=0,82 ; \mathrm{IC}=[(-7,15)-(8,90)]$.

A su vez, se ha comprobado si existen diferencias por edad. Se ha realizado una correlación de Pearson, se ha comprobado que no existen diferencias asociadas a la edad $(r=-0,182, p=0,13)$.

Por último, también se ha analizado si existen diferencias por porcentaje de discapacidad, y se ha realizado una correlación de Pearson. No existe una relación significativa entre ambas $(r=-0,19, p=0,14)$.

\subsection{Análisis de las variables objetivo}

La media del índice total de calidad de vida en puntuación pre fue de 100,82 (DT $=12,39$ ), mientras que en la medida post fue de 111,93 (DT $=10,47$, suponiendo un incremento del 9,92\% en la calidad de vida entre ambas medidas. Una prueba de $t$ de Student para muestras relacionadas (supuesto de normalidad Zpre $=0,783$; Zpost $=0,926)$ muestra que existe una diferencia significativa entre las puntuaciones pre y las post para el índice de calidad de vida $(\mathrm{T}=-5,71 \mathrm{p}<0,001 ; \mathrm{IC}=[(-7,22)-(-5,71)]$ (Figura 2). 
Figura 2. Índice total de calidad de vida en las medidas pre y post

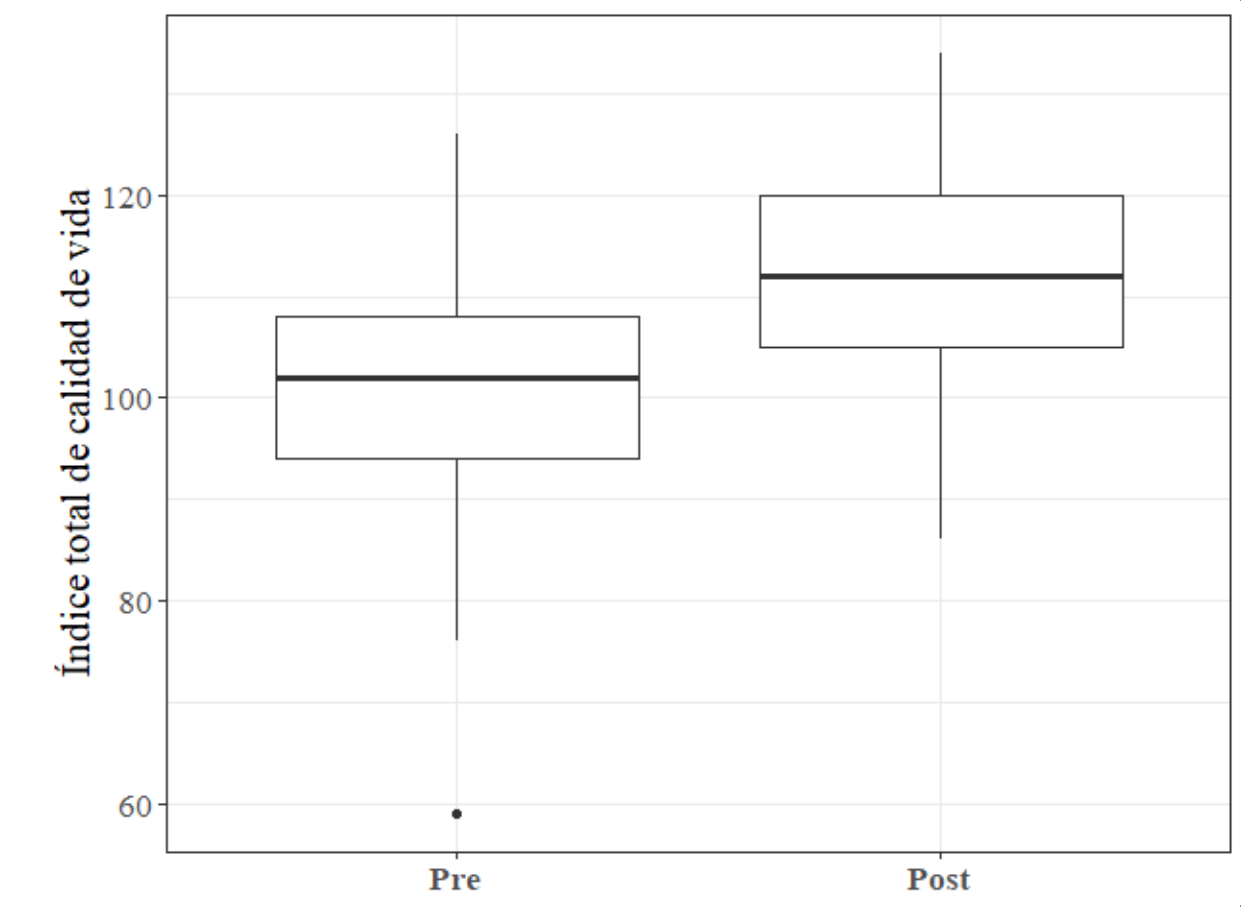

\subsection{Análisis por dimensiones}

Todas las dimensiones se vieron incrementadas en la medida post con respecto a la medida pre, con la dimensión de Derechos siendo la más cambiada $(20,08 \%$ ) y Relaciones interpersonales la que menos (7,35 \%) (Tabla 1). Pruebas de $t$ de Student (y Wilcoxon en su versión robusta) mostraron diferencias significativas entre las medidas pre y post en todas las dimensiones estudiadas (Tabla 1; Figura 3). Se han encontrado tamaños del efecto medianos para Autodeterminación, Bienestar emocional y Relaciones interpersonales y tamaños del efecto grandes para Derechos, Inclusión, Desarrollo personal, Bienestar material y Bienestar físico (Tabla 1). 
IMPACTO DE LA FORMACIÓN PARA EL EMPLEO EN LA CALIDAD DE VIDA

DE JÓVENES CON DISCAPACIDAD INTELECTUAL

S. GARIJO, I. SÁNCHEZ, C. GONZÁLEZ, M. SÁNCHEZ Y A. MARTORELL

\begin{tabular}{|c|c|c|c|c|c|c|c|}
\hline \multirow[b]{2}{*}{ Dimensiones } & \multirow{2}{*}{$\begin{array}{c}\text { Pre } \\
\text { M (DT) }\end{array}$} & \multirow{2}{*}{$\begin{array}{c}\text { Post } \\
\mathrm{M}(\mathrm{DT})\end{array}$} & \multirow{2}{*}{$\begin{array}{c}\text { Porcentaje } \\
\text { de cambio }\end{array}$} & \multicolumn{3}{|c|}{$\mathrm{t}$-Student ${ }^{\mathrm{a}}$} & \multirow{2}{*}{$\begin{array}{r}\text { Tamaño } \\
\text { del efecto }\end{array}$} \\
\hline & & & & $\mathrm{t}$ & IC & $\mathrm{p}$ & \\
\hline Autodeterminación & $\begin{array}{l}11,59 \\
(2,07)\end{array}$ & $\begin{array}{l}12,62 \\
(2,36)\end{array}$ & 8,16 & $-2,83$ & $\begin{array}{c}(-1,76)- \\
(-0,30)\end{array}$ & $0,006^{*}$ & 0,35 \\
\hline Derechos & $\begin{array}{c}9,75 \\
(2,68)\end{array}$ & $\begin{array}{c}12,2 \\
(2,04)\end{array}$ & 20,08 & $-5,74$ & $\begin{array}{c}(-3,29)- \\
(-1,59)\end{array}$ & $<0,001^{*}$ & 1,05 \\
\hline Bienestar emocional & $\begin{array}{c}9,92 \\
(3,06)\end{array}$ & $\begin{array}{l}19,90 \\
(3,00)\end{array}$ & 8,99 & $-2,21$ & $\begin{array}{l}(-2,01) \\
-(0,04)\end{array}$ & $0,027 *$ & 0,48 \\
\hline Inclusión & $\begin{array}{c}9,75 \\
(3,03) \\
\end{array}$ & $\begin{array}{c}11,7 \\
(2,28) \\
\end{array}$ & 16,6 & 3,71 & $\begin{array}{c}(-2,84)- \\
(-1,06)\end{array}$ & $<0,001^{*}$ & 0,78 \\
\hline Desarrollo personal & $\begin{array}{r}9,89 \\
(2,36)\end{array}$ & $\begin{array}{l}11,36 \\
(1,97)\end{array}$ & 12,94 & $-1,47$ & $\begin{array}{c}(-2,29)- \\
(-0,65)\end{array}$ & $0,001 \%$ & 0,89 \\
\hline $\begin{array}{l}\text { Relaciones interper- } \\
\text { sonales }\end{array}$ & $\begin{array}{l}11,21 \\
(2,10)\end{array}$ & $\begin{array}{l}12,10 \\
(2,58)\end{array}$ & 7,35 & $-2,37$ & $\begin{array}{c}(-1,62)- \\
(-0,14)\end{array}$ & $0,018 *$ & 0,5 \\
\hline Bienestar material & $\begin{array}{c}10,2 \\
(2,70)\end{array}$ & $12(2,41)$ & 15 & $-3,76$ & $\begin{array}{c}(-2,66)- \\
(0,94)\end{array}$ & $<0,001^{*}$ & 1 \\
\hline Bienestar físico & $\begin{array}{c}8,77 \\
(3,25)\end{array}$ & $\begin{array}{l}10,08 \\
(2,84)\end{array}$ & 12,99 & $-2,54$ & $\begin{array}{c}(-2,25)- \\
(-0,36)\end{array}$ & $0,011^{*}$ & 0,78 \\
\hline
\end{tabular}

${ }^{a}$ Se utilizó la versión robusta de t-Student (Wilcoxon) en los casos en los que no se cumplía el supuesto de normalidad.

Diferencia significativa con $\mathrm{p}<0.05$.

Ediciones Universidad de Salamanca / CC BY-NC-ND

Siglo Cero, vol. 52 (4), 2021, octubre-diciembre, pp. 51-65 
FIGURA 3. Cambio entre las medidas pre y post para todas las dimensiones
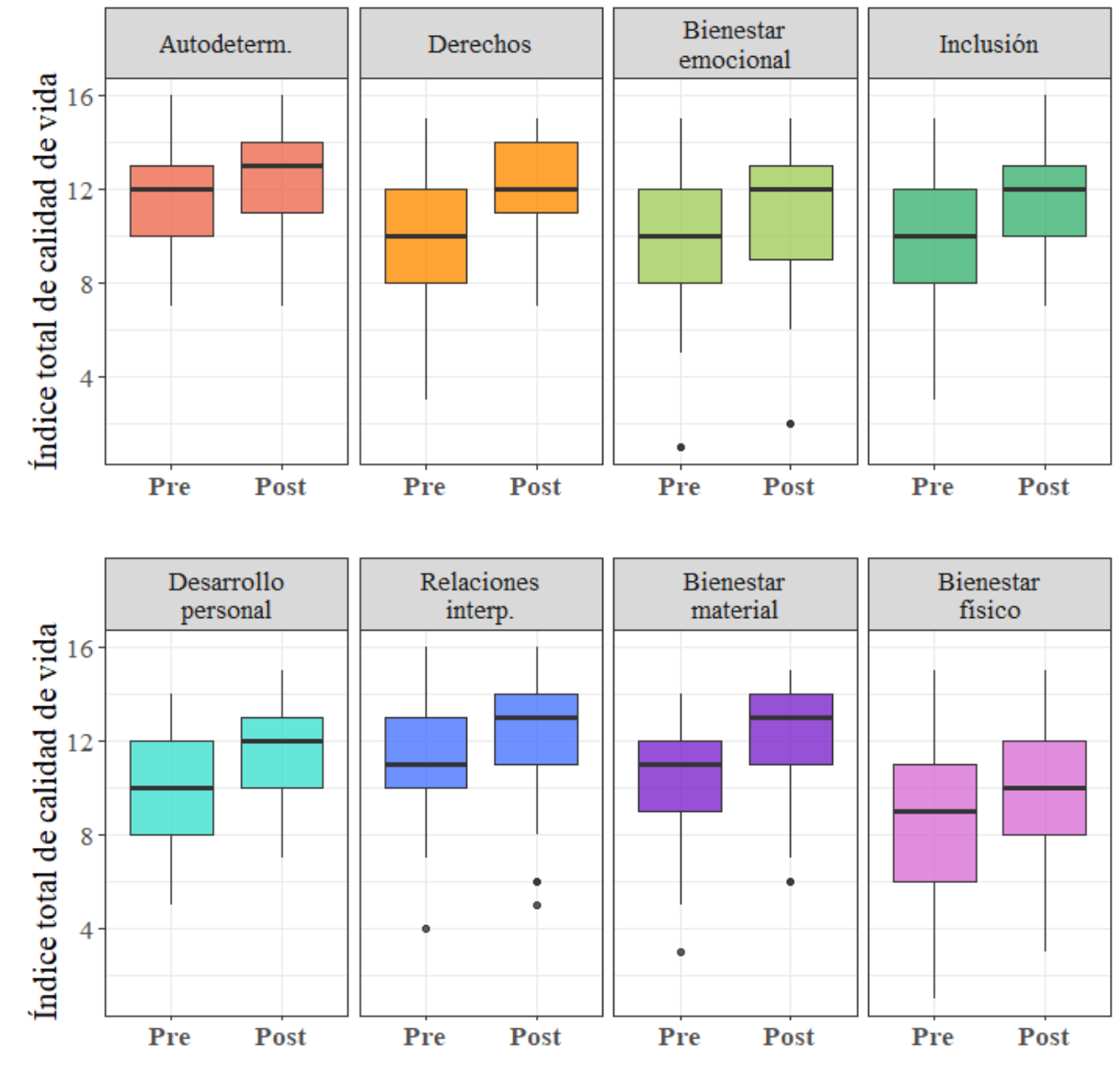

\section{Discusión y conclusiones}

La calidad de vida es un constructo que ha ido ganando importancia y solidez en los últimos años en el ámbito de la investigación de manera global, así como acerca de las personas con discapacidad intelectual.

Cada vez son más los recursos enfocados en mejorar la calidad de vida de las personas con discapacidad intelectual desde diferentes perspectivas (Cabezas y Flórez, 2015). CAMPVS nace como respuesta a una necesidad formativa enfocada a la formación postobligatoria para el empleo para los jóvenes con discapacidad intelectual. Parte de la gran brecha existente entre el empleo dignificante y de calidad y los alumnos con discapacidad intelectual que salen al mundo laboral (Jenaro, 1999). Dada la im- 
portancia de la formación y el empleo en la calidad de vida de un individuo (González Aguilar, 2019; Tobón, 2008), cabe esperar que un programa que aborde tales objetivos mejore tal dimensión.

El proyecto formativo CAMPVS de la Fundación A LA PAR consigue resultados relacionados con el aumento de la calidad de vida de sus alumnos. Los datos de este estudio muestran un aumento significativo en el índice total de calidad de vida, así como en todas sus dimensiones, mostrando unos datos sobre el tamaño del efecto medianos y grandes. Los hallazgos muestran cómo las medidas han aumentado y se han homogeneizado. Tan solo un estudiante ha mostrado efectos que se salen de los esperados, que se han estudiado en detalle y se hipotetiza que se puede deber a sus características particulares en su historia de vida. Estos resultados son similares a los encontrados por Castro et al. (2016) o Izuzquiza Gasset et al. (2013). Además, estos datos no parecen deberse a variables como el género, la edad o el porcentaje de discapacidad, ya que se analizaron de forma previa y no se observó su efecto. Los datos sobre diferencias significativas apoyan las hipótesis planteadas sobre la efectividad del proyecto CAMPVS.

Se puede decir, por tanto, que proyectos orientados a la formación postobligatoria permiten a su alumnado con discapacidad intelectual mejorar su propia calidad de vida, tal y como apuntan estudios previos (Alonso et al., 2018).

Si se analiza con detalle cada una de las dimensiones, se obtiene que no todas aumentan de la misma manera. La dimensión que más ha aumentado de todas las estudiadas es Derechos. Esto puede deberse a la metodología aplicada en el centro formativo, donde el alumno toma un rol protagonista y es empoderado como ciudadano de pleno derecho. Esto se trata en asignaturas tales como Diversidad y Derechos, Orientación Vocacional y Aprendizaje Comunitario, entre otras.

La siguiente dimensión con mayor cambio es Inclusión social. El programa formativo desarrolla su acción en el medio social, es decir, articula una serie de profesionales y servicios tanto dentro como fuera del centro, de forma que el alumnado se siente parte de un grupo, participa en actividades que le gustan, puede ir a diferentes sitios utilizando el transporte público de manera autónoma y recibe apoyo en aquellas cuestiones en las que necesita ayuda. Por ejemplo, el programa de mentoring se desarrolla fuera de las instalaciones del centro con profesionales voluntarios del ámbito de la empresa formados para ser mentores. Estos datos se alinean con estudios realizados recientemente (Morán Suárez et al., 2019).

Las siguientes dimensiones con mayor cambio son Bienestar material y Bienestar físico. En ambos sentidos, el programa no desarrolla ninguna acción concreta dedicada a mejorar tales indicadores, por lo que todo efecto en ellas puede deberse, bien a cambios que sucedan en su vida de manera paralela al programa, o bien, por la toma de consciencia que supone ser un agente activo en sus propios proyectos de vida.

Posteriormente, en cuanto a porcentaje de cambio, encontramos Desarrollo personal, Bienestar emocional y Autodeterminación. Tales indicadores son el eje vertebrador de todas las actividades de CAMPVS y, más concretamente, asignaturas como Habilidades Emocionales, Vida Positiva, Sensibilización y Trabajo Fin de CAMPVS dan forma y contenido a estas dimensiones. Estos datos se soportan en estudios pre- 
vios como el de Arguís et al. (2012) del Programa “Aulas felices” de la Universidad de Zaragoza (Arguís et al., 2012).

Finalmente, la variable que menor cambio porcentual ha conseguido es Relaciones interpersonales. A este respecto, CAMPVS tiene asignaturas en todos los cursos relacionadas con las habilidades que se ponen en práctica en las relaciones interpersonales. Concretamente, se desarrollan asignaturas como Habilidades Sociales, Habilidades Sociolaborales y Búsqueda Activa de Empleo. Además, se ahonda en la importancia de tales relaciones para un desarrollo integral de la persona desde diferentes actividades.

Las dimensiones de Autodeterminación, Bienestar emocional y Relaciones interpersonales son las únicas que tienen un tamaño del efecto moderado en comparación con el resto. Este dato es coherente con lo encontrado en otras investigaciones, en las que los estudiantes con discapacidad tienden a compararse con sus pares sin discapacidad, observando una merma en tales dimensiones (Castro et al., 2016). Para poder ahondar en comprender de qué manera se modifican tales indicadores, se ha decidido abrir nuevas líneas de investigación en el centro relacionadas con las mismas.

Con todos estos datos, se concluye que los jóvenes con discapacidad intelectual, tanto por la ventana temporal en la que actúa CAMPVS como por sus necesidades de apoyo y su permeabilidad a nuevos aprendizajes (Fernández Alcaraz, 2013), aprovechan los recursos formativos para su desarrollo íntegro en términos de calidad de vida.

En definitiva, el programa formativo CAMPVS es una herramienta efectiva para mejorar la calidad de vida de sus estudiantes. De la mano de lo indicado en la Convención Internacional sobre los Derechos de las Personas con Discapacidad (Macho et al., 2012), proyectos como este son necesarios para las personas con discapacidad intelectual. Actualmente, existen muy pocas respuestas adaptadas a las necesidades y potencialidades de los jóvenes con discapacidad intelectual por parte del sistema educativo, y las pocas que hay son iniciativas de índole privada. Los programas de formación se constituyen como un factor fundamental para conseguir una calidad de vida óptima (Alonso et al., 2018).

Se ha de destacar como crítica central a esta investigación que no se puede demostrar que el cambio en la calidad de vida se pueda atribuir al proyecto CAMPVS. El presente estudio es cuasiexperimental y el análisis estadístico realizado nos permite concluir que, después de haber realizado el programa formativo CAMPVS, la calidad de vida de sus estudiantes es mayor a la que encontrábamos antes de que lo comenzasen. Sin embargo, el programa tiene una duración de tres años, y en ese transcurso de tiempo y más en un periodo tan fértil en la vida de los jóvenes con los que se trabaja en el programa (Fernández Alcaraz, 2013), tengan multitud de experiencias que también mejoren su calidad de vida. Asimismo, con el fin de conseguir datos más claros y generalizables, es importante poder conseguir un grupo control de similares características que nos permitiera controlar otras posibles variables, como la edad, los cambios sociales, y así poder aislar el efecto específico del programa CAMPVS en la calidad de vida de sus estudiantes. 
A pesar de que en este estudio se ha medido la posible interferencia del sexo, la edad o el propio porcentaje de discapacidad, con el objetivo de clarificar el efecto del programa y poder aislar el efecto de otras variables, sería bueno monitorizar en futuros estudios las necesidades de apoyo concretas del alumnado y sus habilidades adaptativas con instrumentos validados para tal efecto.

En hilo con lo anterior, se ha de añadir la crítica de que los criterios de selección de CAMPVS en relación con su alumnado hacen que estos datos no puedan ser extendidos a todos los jóvenes con discapacidad intelectual. A pesar de que el porcentaje de discapacidad que aparece en sus informes muestra que en CAMPVS se cuenta con alumnos con discapacidad intelectual con necesidades de apoyo intermitentes o limitadas, en la práctica, al ser un recurso de formación orientada al empleo, tanto por su nivel intelectual como por sus habilidades adaptativas, CAMPVS tiene un perfil muy específico en relación con toda la población de discapacidad intelectual. Es necesaria más investigación donde se incluyan datos con estudiantes con diferentes habilidades intelectuales y de conducta adaptativa.

Se plantean dudas acerca de la variable de Bienestar emocional, dado que el obtenido no es de la magnitud esperada, dado el peso que se le da a lo largo de los años en la formación (Gavín-Chocano y Molero, 2020). Para poder conocer el motivo de este suceso se ha abierto una nueva línea de investigación en el centro con instrumentos exclusivos sobre bienestar.

También sería interesante poder relacionar estos datos de calidad de vida con el nivel de empleabilidad del alumnado, de forma que pudiésemos entender de qué forma afecta tener un grado de calidad de vida para poder tener una mejor inclusión sociolaboral. En este sentido, también se está intentando avanzar en el centro con nuevas líneas de investigación.

En suma con todo lo anterior, es necesaria más investigación que muestre datos sobre proyectos enfocados a mejorar la calidad de vida de los jóvenes con discapacidad intelectual y, sobre todo, sobre cómo incide la formación en dicha calidad de vida, ya que es a través de programas educativos como se puede garantizar el acceso a la sociedad de los jóvenes como ciudadanos plenos, aspecto clave, más aún, cuando hablamos de colectivos vulnerables (González Aguilar, 2019).

Para concluir, en el presente estudio hemos realizado una investigación cuasiexperimental con diseño pre-post con el objetivo de estudiar el impacto de un proyecto de formación para el empleo en la calidad de vida de sus estudiantes con discapacidad intelectual con necesidades de apoyo intermitentes o limitadas. Se concluye que las personas con discapacidad intelectual son sujetos de pleno derecho a poder elegir centros, recursos y proyectos de formación que les ayuden a conseguir los objetivos que son significativos para ellos dentro de su proyecto de vida, entendiendo el empleo como un área especialmente sensible. Se concluye también que tales jóvenes aprovechan tales proyectos para su propio beneficio y que tienen un impacto positivo cuantificable en su calidad de vida, entendiéndola como constructo global y atendiendo a cada una de sus dimensiones. También se concluye que, en tal escenario, el proyecto CAMPVS de la Fundación A LA PAR es un recurso útil y pionero, que atiende a las particularidades de cada estudiante y la generalidad de todos ellos, como participan- 


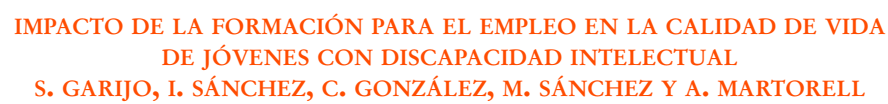

tes activos y protagonistas en sus propias vidas. Y, así mismo, abre las puertas de la esperanza y del optimismo para que se puedan implementar más proyectos por parte de las instituciones, tanto públicas como privadas, que atiendan a las necesidades de los colectivos vulnerables, en búsqueda de una mejor preparación para la vida adulta e inclusión sociolaboral, así como en la garantía de una óptima calidad de vida.

\section{Referencias bibliográficas}

Apa Publications and Communications Board Working Group on Journal Article RePORTING STANDARDs. (2008). Reporting standards for research in psychology. The American Psychologist, 63(9), 839-851. https://doi.org/10.1037/0003-066x.63.9.839

Arguís, R., Bolsas, A. P., Hernández, S. y Salvador, M. del M. (2011). La Psicología Positiva entra en las aulas. Aragón Educa: Revista del Museo Pedagógico de Aragón, 3, 52-57.

Asociación Médica Mundial. (2008). Declaración de Helsinki de la Asociación Médica Mundial. Principios éticos para las investigaciones médicas en seres humanos. Anales del Sistema Sanitario de Navarra, 24(2), 209-212.

Cabezas, D. y Flórez, J. (2015). Educación postsecundaria en entorno universitario para alumnos con discapacidad intelectual: experiencias y resultados. Fundación Iberoamericana Down 21 y Fundación Prodis.

Castro, L., Cerda, G., Vallejos, V., Zúñiga, D. y Cano, R. (2016). Calidad de vida de personas con discapacidad intelectual en centros de formación laboral. Avances en Psicología Latinoamericana, 34(1), 175-186. https://doi.org/10.12804/apl34.1.2016.12

CoHen, J. (2013). Statistical power analysis for the behavioral sciences. Academic Press.

EcheITA, G. y Simón, C. (2014). La contribución de la educación escolar a la calidad de vida de las personas con discapacidad. Ante el desafío de su inclusión social. En R. de Lorenzo y L. CAYo (Eds.), Tratado sobre Discapacidad (pp. 1103-1134). Thomson \& Aranzadi.

Fernández Alcaraz, C. (2013). Estudio longitudinal del perfil neuropsicológico de adultos con discapacidad intelectual con y sin Sindrome de Down. Tesis.

García-Sanjuán, N. y Berástegui, A. (2017). Programa Demos. Formación para jóvenes con discapacidad intelectual en entorno universitario. Integra2 Revista Electrónica de Educación Especial y Familia, 1(8), 39-45.

Gavín-Chocano, Ó. y Molero, D. (2020). Valor predictivo de la inteligencia emocional percibida y calidad de vida sobre la satisfacción vital en personas con discapacidad intelectual. Revista de Investigación Educativa, 38(1), 131-148. https://doi.org/10.6018/rie.331991

Giráldez Hayes, A. y Van Nieuwerburgh, C. (2016). Coaching educativo. Colección: Didáctica y Desarrollo. Ediciones Paraninfo, S. A.

Gómez, M. y SABeh, E. (2000). Calidad de vida. Evolución del concepto y su influencia en la investigación y la práctica, Integra, 9, 3-4.

GonzÁlez Aguilar, J. A. (2019). Intensidad de apoyos, salud mental, empleo y su relación con resultados de calidad de vida. Siglo Cero, 50(2), 73. https://doi.org/10.14201/scero20195027388

HunTER, J. D. (2008). The death of character: moral education in an age without good or evil. Basic Books.

Izuzquiza Gasset, D., Egido Gálvez, I. y Cerrillo Martín, R. (2013). Diez años de formación para el empleo de personas con discapacidad intelectual en la universidad: balance y

Ediciones Universidad de Salamanca / CC BY-NC-ND

Siglo Cero, vol. 52 (4), 2021, octubre-diciembre, pp. 51-65 


\section{IMPACTO DE LA FORMACIÓN PARA EL EMPLEO EN LA CALIDAD DE VIDA DE JÓVENES CON DISCAPACIDAD INTELECTUAL \\ S. GARIJO, I. SÁNCHEZ, C. GONZÁLEZ, M. SÁNCHEZ Y A. MARTORELL}

perspectivas. Revista Iberoamericana de Educación, 63, 127-138. https://doi.org/10.35362/ rie630505

Jenaro, C. (1999). La transición a la vida adulta en jóvenes con discapacidad: Necesidades y demandas. En III Jornadas Científicas de Investigación sobre Personas con Discapacidad.

MorÁn, M. ${ }^{a}$ L., GÓMEZ, L. E. y AlCEDO, M. Á. (2019). Inclusión social y autodeterminación: los retos en la calidad de vida de los jóvenes con autismo y discapacidad intelectual. Siglo Cero, 50(3), 29. https://doi.org/10.14201/scero20195032946

Navas, P., Gómez, L. E., Verdugo, M. Á. y Schalock, R. L. (2012). Derechos de las personas con discapacidad intelectual: implicaciones de la Convención de Naciones Unidas. Siglo Cero, 43(3), 7-28.

Palacios, A. y BarifFi, F. (2007). La discapacidad como una cuestión de derechos humanos: una aproximación a la Convención Internacional sobre los Derechos de las Personas con Discapacidad. Cinca.

Rosete, A. Á., García, L. B., Castillo, A. B., Díaz-Veiga, P., Lantarón, H. G., Navarro, J. A. G., ... Flores, S. L. (2014). Modelo de atención integral y centrada en la persona. Tecnos.

SAMANES, B. E. (2016). Transferencia del sistema de Fp dual a España. Revista de Investigación Educativa, 34(2), 295-314. https://doi.org/10.6018/rie.34.2.249341

SCHALOCK, R. L. (2013). La nueva definición de discapacidad intelectual, apoyos individuales y resultados personales. http://riberdis.cedd.net/handle/11181/3841

Schalock, R. L. y Verdugo, M. Á. (2007). El concepto de calidad de vida en los servicios y apoyos para personas con discapacidad intelectual. Siglo Cero, 38(4), 224, 21-36. https:// gredos.usal.es/handle/10366/55873

TAPIA, M. N. (2006). Aprendizaje y servicio solidario: en el sistema educativo y las organizaciones juveniles. Edición literaria a cargo de: Damián Luis García. Ciudad Nueva.

Тово́N, S. (2008). La formación basada en competencias en la educación superior: el enfoque complejo.

Velázquez, E. D. (2018). La discapacidad en las políticas sociales. En Políticas públicas en tiempos de incertidumbre: aportes para una agenda de investigación (pp. 297-313). https:// dialnet.unirioja.es/servlet/articulo?codigo $=6527801$

Verdugo, M. Á., Amor, A. M., Sánchez, M., Navas, P. y Calvo, M. ${ }^{a}$ I. (2018). La regulación de la inclusión educativa del alumnado con discapacidad intelectual: una reforma pendiente. Siglo Cero, 49(2), 27-58.

Verdugo, M. Á., Arias, B., Santamaría, M. y Clavero, D. (2013). Evaluación de la calidad de vida en personas con discapacidades intelectuales o del desarrollo: la escala INICO-FEAPS. Siglo Cero, 44(247), 6-20. 


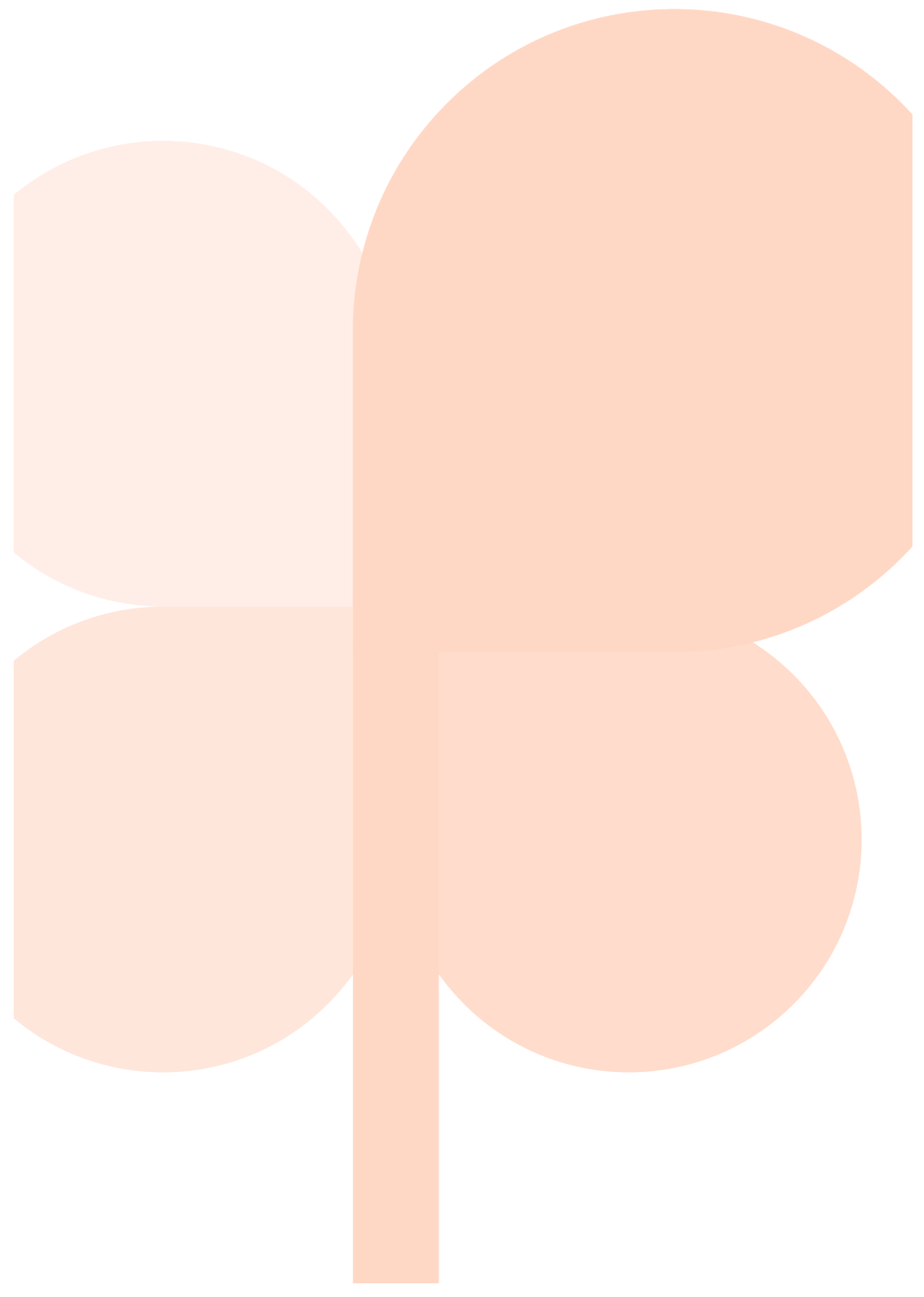

